\title{
Beurteilungs- und Prognosekriterien bei COPD ${ }^{1}$
}

\author{
Assessment and Outcome Parameters in COPD
}

Autoren

Institute
R. Dierkesmann ${ }^{1}$, A. Gillissen ${ }^{2}$, J. Lorenz ${ }^{3}$, H. Magnussen ${ }^{4}$, H. Mitfessel ${ }^{5}$, H. Morr 6 , M. Pfeifer ${ }^{7}$,

G. Schultze-Werninghaus ${ }^{8}$, G. Steinkamp ${ }^{9}$, H. Teschler ${ }^{10}$, Th. Voshaar $^{11}$, T. Welte ${ }^{12}$, H. Worth ${ }^{13}$

Die Institutsangaben sind am Ende des Beitrags gelistet. eingereicht 27.9. 2008

akzeptiert nach Revision

13.10.2008

\section{Bibliografie}

Dol $10.1055 / \mathrm{s}-0028-1100823$ Online-Publikation: 9.1.2009 Pneumologie 2009; 63: 49-55 (C) Georg Thieme Verlag KG Stuttgart · New York ISSN 0934-8387

Korrespondenzadresse

\section{Prof. Dr. med.}

\section{G. Schultze-Werninghaus} Berufsgenossenschaftliches Universitätsklinikum Bergmannsheil GmbH, Medizinische Klinik III Pneumologie, Allergologie, Schlaf- und Beatmungsmedizin Bürkle-de-la-Camp-Platz 1 44789 Bochum gerhard.schultze-werninghaus@bergmannsheil.de

\section{Zusammenfassung \\ $\nabla$}

Hintergrund: Bisher ist für Therapiestudien bei COPD kein einheitlicher Zielparameter definiert. Daher werden in dieser Übersicht häufig verwendete Parameter im Hinblick auf ihre Eignung als Beurteilungs- und Prognosekriterien bei COPD bewertet.

Methodik: Recherche der aktuellen Literatur.

Resultate: Empfohlen wird weiterhin der am besten evaluierte Lungenfunktionsparameter $\mathrm{FEV}_{1}$, ergänzt durch die Messung weiterer Funktionsparameter, wie FVC, IC/TLC und bei entsprechender Ausstattung RV/TLC, $\mathrm{K}_{\mathrm{co}}, \mathrm{PaO}_{2}$ und Pa$\mathrm{CO}_{2}$. Zusätzliche Informationen zur Bestimmung der $\mathrm{FEV}_{1}$ geben die Erfassung von Dyspnoe, Lebensqualität und körperlicher Belastbarkeit, Häufigkeit und Schweregrad von Exazerbationen sowie die Mortalität.

Schlussfolgerung: Bisher wurde keiner der zusätzlichen Parameter so gut evaluiert, dass er derzeit als Ersatz für die FEV $_{1}$ als Standardparameter für die Beurteilung und Prognose der COPD dienen könnte.

\section{Einleitung und Methodik \\ $\nabla$}

Die Resultate von klinischen Prüfungen zur Pharmakotherapie der COPD sind abhängig von den gewählten Ein- und Ausschlusskriterien und von den Zielparametern. Die im vergangenen Jahrzehnt publizierten Studien hatten uneinheitliche Ergebnisse, nicht zuletzt aufgrund unterschiedlicher Beurteilungskriterien [1]. In dieser Übersichtsarbeit werden daher verschiedene Zielparameter in Ergänzung zu ähnlichen Publikationen aus neuerer Zeit auf ihre Eignung hin überprüft $[2,3]$, beruhend auf einer Recherche der aktuellen Literatur.

\section{Abstract \\ $\nabla$}

Background: A standard outcome parameter for pharmacological trials in COPD has not yet been defined. Therefore, it is the aim of this review to evaluate frequently used parameters for their eligibility as assessment and outcome parameters in COPD.

Methods: A review of the actual scientific literature was performed.

Results: It is recommended to continue to rely primarily on the $\mathrm{FEV}_{1}$, which has been used as a primary variable in the vast majority of trials. In addition, further parameters, such as FVC and IC/ TLC should be determined. If available, additional information is provided by $\mathrm{RV} / \mathrm{TLC}, \mathrm{K}_{\mathrm{co}}, \mathrm{PaO}_{2}$ and $\mathrm{PaCO}_{2}$. $\mathrm{FEV}_{1}$ is not a surrogate parameter for dyspnoea, quality of life, and exercise tolerance, which should therefore be assessed separately. Frequency and severity of exacerbations and mortality are important outcome parameters in long-term trials. Complex indices, such as the BODE index, may be superior to single variables. Conclusions: No single additional parameter has been evaluated sufficiently in order to substitute $\mathrm{FEV}_{1}$ as the standard parameter for the assessment and outcome in COPD.

\section{Ergebnisse}

$\nabla$

\section{Lungenfunktion}

Obstruktionsparameter, Reversibilität, CO-Diffusion, 6-min-Gehtest, Compliance

Die $\mathrm{FEV}_{1}$ ist der am besten evaluierte Parameter zur Quantifizierung einer Atemwegsobstruktion [4]. Nach internationalen und nationalen Leitlinien stützt sich der Nachweis einer COPD auf den Schwellenwert der $\mathrm{FEV}_{1} / \mathrm{FVC}<70 \%$ und die

${ }^{1}$ Ergebnisse des Expertentreffens "Luftschlösser“, 23. - 24. November 2007, Frankfurt am Main, mit Unterstützung der Boehringer Ingelheim Pharma GmbH \& Co KG 
Schweregradeinteilung insbesondere auf die $\mathrm{FEV}_{1}$, angegeben in Prozent des Sollwerts [5]. Allerdings eignen sich Schwellenwerte für Lungenfunktionsparameter wegen der altersbedingten Variabilität der Lungenfunktion nur eingeschränkt für die Definition des COPD-Schweregrades, denn eine $\mathrm{FEV}_{1} / \mathrm{FVC}<70 \%$ des Solls beobachtet man teilweise auch bei gesunden Menschen über 65 Jahre. Daher wurde schon in den 90er-Jahren eine altersbezogene Flexibilität der Messwerte empfohlen („lower limit of normal“, LLN), später aber wieder verworfen [6]. Ferner wurden die Schwellenwerte bisher nicht validiert [5].

Die GOLD-Leitlinien stützen sich bei der Beurteilung der $\mathrm{FEV}_{1}$ auf den Messwert nach Bronchodilatation, mit dem Ziel einer geringeren Variabilität der Resultate. Es ist nicht validiert, ob diese Auffassung zutrifft. Das Testergebnis schwankt im Krankheitsverlauf [7] und in Abhängigkeit von den technischen Bedingungen. Neuerdings wird die post-bronchodilatatorisch gemessene $\mathrm{FEV}_{6}$ (forciertes exspiratorisches Volumen in 6 Sekunden) als weiterer brauchbarer Restriktionsparameter statt der bei Obstruktion nicht immer exakt bestimmbaren FVC diskutiert, ist aber nach neueren Daten der FVC doch nicht überlegen [8].

Das forcierte inspiratorische Volumen $\left(\right.$ FIV $\left._{1}\right)$ und andere inspiratorische Parameter (z.B. FIVC) bieten sich gegenüber den exspiratorischen Parametern als geeignetere Parameter zur Quantifizierung der Lungenfunktion bei COPD-Patienten an [9]. Die inspiratorisch gemessenen Volumina liegen bei COPD über den der entsprechenden exspiratorischen Werte. Da das prozentuale Verhältnis etwaiger Veränderungen aber bei beiden Messmethoden vergleichbar ist, ergibt sich z.B. zur Abschätzung einer Therapiemaßnahme kein signifikanter Vorteil der inspiratorischen Messwerte.

Das Ausmaß der CO-Diffusionskapazitäts-Einschränkung $\left(\mathrm{D}_{\mathrm{L}, \mathrm{Co}}\right)$ weist auf die Schwere eines Lungenemphysems hin; wie eine vergleichende Studie mit HRCT-Untersuchungen zeigte, kann diese aber in der Emphysemdiagnostik nicht ersetzen, zumal die $D_{L, C O}$ diversen Störgrößen unterworfen ist [10]. Auch der 6-Minuten-Gehtest und die maximale physische Leistungsbreite korrelierten bei schwerer Einschränkung gut mit den $\mathrm{D}_{\mathrm{L}, \mathrm{CO}}-\mathrm{Be}-$ funden. Sauerstoffpflichtige Patienten hatten z.B. in einem hohen Prozentsatz auch eine pathologische $\mathrm{D}_{\mathrm{L}, \mathrm{Co}}[11]$.

Die Bestimmung der Lungendehnbarkeit (Compliance) ist eine wertvolle Ergänzung der Lungenfunktionsprüfung und geeignet, bei Patienten mit erniedrigter CO-Diffusion zwischen Lungenfibrose und Emphysem zu differenzieren [12]. Allerdings ist die Untersuchung für den Patienten aufwändig und belastend, denn für die Registrierung der Druck-Volumen-Kurve zur Messung der Lungencompliance ist die intrathorakale Druckmessung mittels eines Ösophagus-Katheters notwendig. Aufgrund ihres technischen Aufwandes wird die Compliancemessung nur für wissenschaftliche Fragestellungen eingesetzt.

\section{Überblähungsparameter}

Bei COPD-Patienten ist die Lungenüberblähung neben der Atemwegsobstruktion ein wesentlicher Grund für die Dyspnoe. Die Lungenüberblähung führt zur Abflachung und zum Tiefstand des Zwerchfells, konsekutiv zu einer zunehmenden Einschränkung der Atemmechanik bzw. der „Atempumpe“ infolge eines Funktionsverlustes des wichtigsten Atemmuskels. Die Atemmittellage verschiebt sich zur Inspiration hin in Richtung der totalen Lungenkapazität TLC, und die inspiratorische Kapazität IC bzw. die Vitalkapazität - IVC, FVC - nehmen infolge der erhöhten funktionellen Residualkapazität FRC bzw. des erhöhten intrathorakalen Gasvolumens ITGV ab. Eine solche Überblähung der
Lunge ist zwar nicht zwingend an die Ausbildung eines Emphysems gebunden. Meistens ist jedoch eine nicht-reversible Lungenüberblähung durch eine irreversible Parenchymdestruktion bedingt. Unter Belastung kann auch ohne Emphysem eine dynamische Überblähung auftreten, die die Belastungsfähigkeit der Patienten entscheidend einschränkt.

Funktionsparameter können genutzt werden, um eine Überblähung zu quantifizieren [3]. Die sichere Bestimmung der Überblähung erfordert die Kombination von Ganzkörperplethysmografie und Spirometrie bzw. die Bestimmung der funktionellen Residualkapazität (FRC) mittels Helium. Das endexspiratorische Lungenvolumen (EELV), die funktionelle Residualkapazität (FRC/ ITGV) und das Residualvolumen (RV) sind erhöht, als indirekte Parameter sind die inspiratorische Kapazität (IC) und die Vitalkapazität $\left(\mathrm{VC}_{\max }\right)$ erniedrigt. Eine Diskrepanz zwischen der Bestimmung des FRC mittels Helium bzw. Ganzkörperplethysmografie gibt Hinweise auf den irreversiblen, emphysembedingten Anteil der Lungenüberblähung. Ein Volumenbezug der Überblähungsparameter auf die TLC ist erforderlich. Zur Quantifizierung der Überblähung eignen sich in der Routine besonders die Parameter RV/TLC und ITGV/TLC. Der Quotient IC/TLC gilt als signifikanter Prädiktor der Mortalität bei COPD [3]. Zeigt die Fluss-Volumen-Kurve einen exspiratorischen Kollaps, so weist dies auf eine dynamische Überblähung hin. Ursache sind vorwiegend irreversible strukturelle Änderungen von Lunge und Atemwegen mit einem Verlust der elastischen Rückstellkräfte des Gewebes. Die Lungenüberblähung lässt sich medikamentös reduzieren, insbesondere mit Tiotropium [13], so dass eine Erfassung der Überblähungsparameter wichtige zusätzliche Erkenntnisse vermittelt.

\section{Blutgase, Belastungstests, Atempumpe}

Ein $\mathrm{PaCO}_{2}$-Anstieg im Sinne einer ventilatorischen Insuffizienz korreliert mit dem Ausmaß der $\mathrm{FEV}_{1}$-Einschränkung [14]. Der $\mathrm{PaO}_{2}$ während der Nacht ist mit der Überlebensprognose assoziiert: Patienten, bei denen während des REM-Schlafes ein relevanter Sauerstoffabfall eintritt, leben signifikant kürzer [15]. Diese Patientengruppe profitiert auch am stärksten von einer Sauerstofftherapie. Eine Differenzierung der Ursachen einer nächtlichen Hypoxämie ist notwendig, um eine obstruktive Schlaf-Apnoe von einer COPD-assoziierten Hypoventilation abzugrenzen und ggf. jede Erkrankung angemessen zu therapieren. Während eines Belastungstests kann ein Abfall des $\mathrm{PaO}_{2}$ verschiedene Gründe haben: ein Missverhältnis zwischen Ventilation und Perfusion, eine reduzierte Gasaustauschfläche, Obstruktion/Überblähung oder muskuläre Dekonditionierung. Die beiden letztgenannten Ursachen gehen mit einem $\mathrm{PaCO}_{2}$-Anstieg bei zunehmender Belastung einher (ventilatorische Insuffizienz).

Veränderungen nach einer medikamentösen Behandlung lassen sich mit den Parametern Belastungsdauer (endurance time) und maximale Sauerstoffaufnahme erfassen [16]. So stieg nach sechswöchiger Behandlung mit Tiotropium die Belastungsdauer um $105+40$ s (21\%) im Vergleich zu Plazebo [13]

Die Spiroergometrie bildet verschiedene Dimensionen ab, wie Atemmechanik, Hämodynamik, muskuläre Funktion und Stoffwechselkapazität. Die maximale Sauerstoffaufnahme korreliert mit der Überlebensprognose männlicher COPD-Patienten [17]. Ein anderer signifikanter prognostischer Parameter ist die Geschwindigkeit, mit der die belastungsinduzierte Hypoxämie auftritt $\left(\mathrm{PaO}_{2}\right.$ slope [18]). 
Sowohl die Zwerchfell- als auch die Skelettmuskulatur ist bei COPD erkrankt: der maximale exspiratorische Druck, der die generalisierte Muskelkraft repräsentiert, ist bei COPD erniedrigt, ebenso wie der maximale inspiratorische Druck, der die Zwerchfellfunktion und -Mechanik reflektiert [19,20].

\section{Pulmonale Hypertonie}

Eine pulmonale Hypertonie wird durch eine COPD ohne Begleiterkrankungen nicht regelhaft verursacht [21]. Leichte bis mittelgradige Erhöhungen des mittleren Pulmonalarteriendrucks (PAP) auf $>20 \mathrm{~mm} \mathrm{Hg}$ wurden bei $20-35 \%$ der COPD-Patienten gefunden, während Drucke $>40 \mathrm{~mm} \mathrm{Hg}$ mit nur $7,5 \%$ deutlich seltener waren [22]. Von 131 COPD-Patienten, die in Ruhe und unter Belastung untersucht wurden, hatten 55 weder in Ruhe noch unter Belastung PAP-Werte $>20 \mathrm{~mm} \mathrm{Hg}$ [23]. Eine relevante pulmonale Hypertonie beeinflusst die Überlebensprognose von COPD-Patienten ungünstig [24] und ist mit häufigeren Exazerbationen assoziiert [25].

Um die Diagnose einer pulmonalen Hypertonie zu sichern, ist eine invasive Messung erforderlich. Mit der Echokardiografie erhält man keine verlässlichen Ergebnisse. Während Parameter wie $\mathrm{FEV}_{1}, \mathrm{D}_{\mathrm{L}, \mathrm{Co}}$ oder CT-Score nur schwach mit der Schwere der pulmonalen Hypertonie korrelierten, bestand in einer aktuellen Untersuchung eine signifikante Korrelation zwischen PAP und $\mathrm{PaO}_{2}[26]$.

Bei der Entstehung der pulmonalen Hypertonie ist nicht nur die hypoxämisch bedingte Druckerhöhung im Sinne des Euler-Liljestrand-Mechanismus von Bedeutung. Anatomische Faktoren treten hinzu, insbesondere die Verringerung des Gefäßquerschnitts. Außerdem kommt es zu Umbauvorgängen in den Blutgefäßen. Ursächlich liegen diesem vaskulären Remodelling systemische Entzündungsprozesse zu Grunde. Durch die Einwirkung von Zigarettenrauch wird das Endothel geschädigt und eine Proliferation von Gefäßzellen ausgelöst. Hyperkapnie und Azidose bewirken eine renale Salz- und Wasserretention und damit eine Flüssigkeitsbelastung. Die genannten Veränderungen bewirken zwar einen Druckanstieg im kleinen Kreislauf, dieser ist jedoch von der Ausprägung her allenfalls mittelgradig. Für die Entwicklung einer schweren pulmonalen Hypertonie sind zusätzliche Faktoren erforderlich. Meist muss dabei von einer eigenständigen Erkrankung ausgegangen werden, mit entscheidenden therapeutischen Konsequenzen. Während eine spezifische Therapie der COPD-assoziierten mäßigen Erhöhung des pulmonal-vaskulären Druckes nicht gesichert ist, besteht bei einer eigenständigen pulmonalen Hypertonie die Indikation zur Therapie mit modernen Vasodilatatoren.

\section{COPD und Schlaf}

Bei COPD sind Häufigkeit und Ausmaß von nächtlicher Hypoxie und Hyperkapnie abhängig vom klinischen Phänotyp und vom Schweregrad der COPD. Im Vergleich zu Patienten vom Typ „Pink Puffer“ sind „Blue Bloater“ weitaus stärker betroffen: Ihre Zwerchfellfunktion ist gestört, und die Sauerstoffsättigung kann während der Nacht um bis zu 30\% abfallen [27]. Eine Verschlechterung der Oxygenierung im Schlaf kommt bei einer $\mathrm{FEV}_{1}$ unter $60 \%$ des Solls zwar häufiger vor [28], eine verlässliche Vorhersage der nächtlichen Gasaustauschstörung anhand von Lungenfunktionsparametern oder der Sauerstoffsättigung im Wachzustand ist jedoch nicht möglich. Bei COPD-Patienten mit einer mittleren $\mathrm{FEV}_{1}$ von $32 \%$ des Solls verbesserte Tiotropium die Sauerstoffsättigung im Schlaf, ohne die Schlafqualität zu verändern [29].
Komorbiditäten wie die obstruktive Schlafapnoe (OSA) haben einen entscheidenden Einfluss auf das Ausmaß der nächtlichen Gasaustauschstörung, und sie liefern eine plausible Interpretation für die Unterschiede zwischen „Pink Puffern“ und „Blue Bloatern“ [27]. Von den OSA-Betroffenen haben 9-16\% gleichzeitig eine COPD, und umgekehrt findet man bei $6-14 \%$ der COPD-Patienten gleichzeitig eine OSA. Dieses so genannte „Overlap-Syndrom" trägt zur nächtlichen Hypoventilation/Hypoxie ebenso bei wie zur Entwicklung einer respiratorischen Globalinsuffizienz. Die kardiovaskuläre Mortalität ist am höchsten bei Männern mit schwerer OSA, d.h. mit einem Apnoe-Hypopnoe-Index $>30 / \mathrm{h} \mathrm{[30]}$. In einer Fall-Kontrollstudie bei Männern mit OSA war die COPD der stärkste Prädiktor für Mortalität, gefolgt von chronischer Herzinsuffizienz, Diabetes mellitus und einem erhöhten Body Mass-Index [31]. Dementsprechend erscheint bei COPD-Patienten mit Schweregrad GOLD III oder IV eine Polygrafie gerechtfertigt.

\section{Dyspnoe}

Atemnot ist ein subjektives Empfinden und individuell sehr unterschiedlich ausgeprägt. Die Einschätzung von Patienten wird in verschiedenen Dyspnoe-Skalen abgebildet. Am bekanntesten ist die Medical Research Council Skala (MRC), die auch Bestandteil des BODE-Index geworden ist. Häufig verwendet werden auch Baseline und Transition Dyspnoe Indices (BDI, TDI) und die Dyspnoe-Domäne des Chronic Respiratory Disease Questionnaire (CRQ). Für die Einschätzung von Dyspnoe während körperlicher Belastung eignen sich die Borg-Skala oder die Visual Analog Skala (VAS). Dyspnoe-Skalen korrelieren nur bedingt mit anderen Krankheitsparametern [32], fanden keine Korrelation zwischen Dyspnoe und Veränderung der $\mathrm{FEV}_{1}$, jedoch Assoziationen zur FIV ${ }_{1}$. Bei stabiler COPD korrelierte die Dyspnoe nicht mit der $\mathrm{FEV}_{1}$, jedoch mit der Lebensqualität, gemessen mit dem SGRQ [33].

In Medikamentenprüfungen verringerte sich die Dyspnoe nach Tiotropium signifikant stärker als nach Ipratropium oder nach Plazebo $[34,35]$. Auch für Salmeterol wurde eine günstige Beeinflussung der Atemnot nachgewiesen [36]. In einer vergleichenden Studie besserten sich COPD-Patienten, die inhalative Steroide erhielten, in ihrem mittleren TDI-Gesamtscore unter Tiotropium stärker als unter Salmeterol oder Plazebo [37].

\section{Lebensqualität}

Zur Messung der Lebensqualität bei COPD eignen sich vor allem krankheitsspezifische Fragebögen, wie der St. George's Respiratory Questionnaire (SGRQ). Hier gelten bisher Abnahmen um mindestens 4 Punkte als relevante Verbesserungen für den Patientenalltag $[3,38]$. Signifikant stärker als unter Plazebo verbesserte sich die Lebensqualität nach Tiotropium [37], nach der Budesonid-Formoterol-Fixkombination [39] und nach der Salmeterol-Fluticason-Fixkombination [40]. Die Kombination antiobstruktiver Medikamente wurde in einer kürzlich publizierten Studie überprüft [41]. Der primäre Zielparameter, die Zahl der Patienten mit Exazerbationen, unterschied sich nicht bei der Behandlung mit Tiotropium plus Plazebo, Tiotropium plus Salmeterol oder Tiotropium plus Salmeterol-Fluticason. Jedoch zeigte die letztgenannte Patientengruppe signifikant bessere Ergebnisse für die Lebensqualität, neben den Parametern Lungenfunktion und Krankenhausaufnahmen.

In COPD-Studien war die Lebensqualität bisher nur ein sekundäres Zielkriterium. Das Institut für Qualität und Wirtschaftlichkeit im Gesundheitswesen, nicht aber FDA oder EMEA, sieht die 
Lebensqualität als wichtigen Parameter für die Beurteilung der Arzneimittelwirksamkeit an. Daher sollte die Lebensqualität als primärer Endpunkt in zukünftigen klinischen Prüfungen erfasst werden. In der Praxis spielen die genannten Scores nur eine untergeordnete Rolle, da praktisch alle zu komplex sind, um in den Praxisalltag integriert werden zu können.

\section{Exazerbationsraten}

Zur Definition der COPD-Exazerbation werden einerseits klinische Symptome herangezogen, wie Zunahme von Husten, Sputum und Dyspnoe, andererseits bezieht man sich auf Ereignisse, wie die Änderung der Medikation oder den Besuch beim Arzt. Bisher fehlt ein Konsens darüber, ob eine Exazerbation mehr Ereignis- oder mehr Symptom-orientiert zu verstehen ist. Der Patient nimmt eine Exazerbation nicht immer wahr. Dies ist aber die Voraussetzung dafür, dass seine Behandlung intensiviert werden kann. Nicht registrierte/wahrgenommene Exazerbationen waren in einer aktuellen Untersuchung genauso häufig wie registrierte [42]. Hier könnte ein Patiententagebuch helfen, die Symptome genauer zu erfassen. Für die Festlegung der Therapie spielt der Schweregrad einer Exazerbation eine wichtige Rolle, wie er z. B. von Burge [43] definiert wurde. Wie eine Auswertung von 22 Studien mit mehr als 17000 Patienten zeigte, bestehen bezüglich der statistischen Auswertung von Studienergebnissen zahlreiche Probleme [44]. Dazu gehört, dass Patienten nicht vollständig analysiert werden, die eine Studie vorzeitig beendet haben.

Häufigkeit und Qualität von Exazerbationen haben einen gesicherten Einfluss auf die Mortalität der COPD. Sie sind mit einem schlechteren BODE-Index assoziiert [45] und beeinflussen signifikant das Überleben [46]. Risikofaktoren für Exazerbationen und Krankenhausaufnahmen bei Männern mit COPD wurden von Niewoehner u. Mitarb. prospektiv untersucht [47]. Höheres Alter, niedrige $\mathrm{FEV}_{1}$ und Klinikbehandlung im letzten Jahr waren mit beiden Ereignissen assoziiert. Produktiver Husten, Antibiotika- oder Steroidtherapie waren Risikofaktoren für Exazerbationen, während kardiovaskuläre Erkrankungen oder Notfallbesuche beim Arzt Prädiktoren für eine Hospitalisierung waren. Die multivariate statistische Analyse mündete in der Berechnung eines Risikoscores für eine Exazerbation in den kommenden 6 Monaten (7 Kriterien, maximal 500 Punkte). Für spezielle Fragen mag es sinnvoll sein, diese Punktzahl zu ermitteln. Medikamente der COPD-Dauertherapie können die Häufigkeit von Exazerbationen verringern. Für Tiotropium ergaben sich Reduktionen der Exazerbationsraten, bei allerdings unterschiedlichen Definitionen, über 6-12 Monate um 19-52\%.

\section{Mortalität}

Angaben zur Mortalität in der Literatur sind nicht immer eindeutig zu interpretieren, da die Datenerfassung auf subjektiven Einschätzungen der bescheinigenden Ärzte beruht. In der BRD lag im Jahr 1999 das mediane Sterbealter von Männern mit COPD bei 76,3 Jahren und war damit 3 Jahre höher als bei der übrigen männlichen Bevölkerung. Dies darf nicht dahingehend fehlinterpretiert werden, dass die COPD einen Schutzfaktor darstellt. Denn nach neueren Untersuchungen sinkt die Überlebenswahrscheinlichkeit erheblich, wenn eine COPD vorliegt, und insbesondere Frauen mit COPD sterben deutlich früher [48].

Vielmehr ist die Kodierung der Mortalitätsursache häufig ungenau. In Totenscheinen aus England und Wales wurde die COPD nur halb so häufig als primäre Todesursache genannt wie als weitere Diagnose [49]. Nach der dänischen Kohortenstudie star- ben $45 \%$ der COPD-Patienten an der COPD, $22 \%$ an kardiovaskulären Erkrankungen und $11 \%$ an einem Bronchialkarzinom [50]. Bei einem relevanten Anteil von $26 \%$ der Patienten war die Todesursache unbekannt, besonders dann, wenn der Tod im Schlaf eintrat [51]. Pneumonien bzw. pulmonale Infektionen machen etwa $10 \%$ der Todesursachen bei COPD aus, vor allem bei schwerer COPD. Weitere wichtige Todesursachen sind Lungenembolie und Diabetes mellitus [52]. Ein Teil der Komorbidität lässt sich durch Alter und Tabakrauchen erklären.

Ebenfalls schwierig zu interpretieren ist der Zusammenhang zwischen Pharmakotherapie und COPD-Morbidität. So war in einer epidemiologischen Studie das Risiko für eine Hospitalisation signifikant höher, wenn Patienten medikamentös behandelt worden waren [53]. Hier kann man die Pharmakotherapie als Indikator für den Schweregrad der Erkrankung auffassen. Inhalative Steroide reduzierten bei COPD nach einer post hoc Analyse von Sin [54] Todesfälle an kardiovaskulären oder malignen Erkrankungen. In einer 3-jährigen Studie war die All Cause Mortality unter inhalativen Steroiden allerdings leicht erhöht [55]. Nach Tiotropium ergab sich ein geringeres relatives Risiko, an kardiovaskulären oder pulmonalen Ursachen oder an einer Pneumonie zu versterben [56]. Generell sollten bei der COPD keine Medikamente verordnet werden, die für die Komorbidität ungünstig sind.

\section{Konsequenzen der systemischen Effekte der COPD}

In den letzten Jahren wurde die COPD als Systemerkrankung begriffen. Mit dem Ziel, einen integrativen Parameter zu entwickeln, entstand der BODE-Index mit den Kriterien Body Mass-Index, Obstruktion, Dyspnoe und Belastbarkeit [57]. Er eignete sich besser als die FEV $_{1}$ oder der Quotient IC/TLC zur Vorhersage der Mortalität [58].

In der TORCH-Studie waren nur 36\% der Todesfälle durch eine pulmonale Erkrankung bedingt, während Herzerkrankungen (27\%) und Tumoren (22\%) weitere wichtige Todesursachen waren [55].

Umgekehrt beeinflusst die Komorbidität COPD den Verlauf anderer Erkrankungen erheblich: alle wichtigen Krankheiten, die zur Hospitalisation führen, sind ernster zu nehmen, wenn der Patient gleichzeitig eine COPD hat [59]. Eine aktuelle Analyse zum Zusammenhang zwischen Raucherstatus, kardiovaskulärer Mortalität und $\mathrm{FEV}_{1}$ ergab zwar bei Rauchern in jeder $\mathrm{FEV}_{1}$-Schweregrad-Gruppe ein erhöhtes Risiko [60]. Jedoch hatten Nichtraucher mit einer $\mathrm{FEV}_{1}$ unter $80 \%$ des Solls ein größeres Risiko als Raucher mit normaler $\mathrm{FEV}_{1}$. Demnach ist die $\mathrm{FEV}_{1}$ als Marker für die globale Mortalität anzusehen.

Regelmäßige körperliche Aktivität reduziert bei COPD sowohl die Hospitalisationsrate als auch die Mortalität [61]. Um das Ausmaß der körperlichen Aktivität als globalen Parameter genauer zu evaluieren, erfasste eine Studie aus Großhansdorf mithilfe des SenseWea ${ }^{\circledR}$ Pro Armbands die Zeit körperlicher Aktivität, die Schrittzahl und den Energieverbrauch [62]. Sowohl die Schrittzahl als auch die Dauer der täglichen Bewegungsaktivität verringerten sich mit zunehmendem GOLD-Schweregrad. Patienten mit Zeichen der systemischen Entzündung (Fibrinogen $>436 \mathrm{mg} / \mathrm{dl}$ ) hatten in den jeweiligen GOLD-Stadien eine geringere körperliche Aktivität als solche mit normalen Werten. Ähnlich war die Situation bei Patienten mit Hinweis auf linksventrikuläre Dysfunktion (NT-pro-BNP > 67,3 pg/ml). Um Patienten zu vermehrter körperlicher Aktivität zu ermuntern, reichen relativ einfache Interventionen aus, wie regelmäßige Telefonanrufe beim Patienten zu Haus [63]. 


\section{Welche Parameter werden von den aktuellen COPD-Leitlinien besonders beriicksichtigt? \\ $\nabla$}

Als aktuelle Leitlinien sind von besonderer Bedeutung 1. die GOLD-Leitlinie [5], 2. die COPD-Leitlinie der Deutschen Atemwegsliga und der Deutschen Gesellschaft für Pneumologie und Beatmungsmedizin [64] und 3. die Nationale Versorgungsleitlinie COPD [65]. Für die Diagnosestellung und zur Einschätzung des Schweregrades der stabilen COPD verwenden alle Leitlinien die (postbronchodilatatorische) $\mathrm{FEV}_{1}$ bzw. die Relation $\mathrm{FEV}_{1} / \mathrm{VC}$. Die DGP-Leitlinie berücksichtigt außerdem das Emphysem mit den Kenngrößen FRC und CO-Diffusionskapazität $\mathrm{D}_{\mathrm{L}, \mathrm{co}}$.

Für den Wirksamkeitsnachweis bestimmter Therapieoptionen eignen sich u. a. die Parameter $\mathrm{FEV}_{1}$, Überblähung, Dyspnoe, Lebensqualität oder Häufigkeit von Exazerbationen. Nicht nur durch Medikamente, sondern auch durch pulmonale Rehabilitation lassen sich diesbezüglich Verbesserungen erreichen. Gute Effekte zeigten sich auch durch Kombination verschiedener Therapieoptionen, wie körperliches Training plus Tiotropium-Medikation [66].

Die an den Schweregrad der COPD angepasste Therapie unterscheidet sich kaum zwischen den Leitlinien. Lang wirksame Bronchodilatatoren und Rehabilitation werden ab Grad II empfohlen, inhalative Steroide bei wiederkehrenden Exazerbationen ab Grad III, und bei sehr schwerer COPD (Schweregrad IV) kommen Langzeitsauerstofftherapie und chirurgische Eingriffe in Betracht. Exazerbationen sind charakterisiert durch vermehrte Symptome und auffällige Blutgase. Die Notwendigkeit einer nicht-invasiven Beatmung wird an den Blutgasen festgemacht $\left(\mathrm{pH}<7,35, \mathrm{PaCO}_{2}>45\right.$ oder $\left.50 \mathrm{~mm} \mathrm{Hg}\right)$.

Unter suffizienter medikamentöser Dauertherapie der COPD konnten respiratorische Exazerbationen um 20-30\% reduziert werden [67]. Eine strukturierte Patientenschulung zeigte diesbezüglich ebenfalls gute Effekte [68].

Leitlinien sind ein wichtiges Instrument des Qualitätsmanagements. Sie müssen den regionalen Gegebenheiten angepasst und in die Alltagsarbeit implementiert werden. Die Versorgungsforschung sollte Qualitätsindikatoren definieren und nachweisen, dass sich die Patientengesundheit durch Anwendung von Leitlinien verbessern lässt.

\section{Beurteilungs- und Prognosekriterien: Was ist in der Praxis machbar? \\ $\nabla$}

Die Mehrzahl der COPD-Patienten wird vom Hausarzt behandelt: In den Niederlanden waren es 1996 85\% [69]. Patienten mit COPD werden häufig falsch oder gar nicht diagnostiziert. Hausärzte und Pneumologen unterscheiden sich darin, welche diagnostischen Kriterien sie benutzen [70]. Pneumologen verlassen sich mehr auf die Lungenfunktion, während Hausärzte in stärkerem Maße die klinischen Symptome heranziehen. Die $\mathrm{FEV}_{1}$ ist wichtig für Diagnosestellung, Schweregradeinteilung und Prognoseeinschätzung. Die Überlebensrate ließ sich mit dem 5-Punkte-MRC-Dyspnoe-Score besser vorhersagen als mit der FEV 1 [71]. Die Borg-Skala mit 10 Punkten hatte eine noch bessere Sensitivität als der MRC-Score [72].

Der 6-Minuten-Gehtest, ein Bestandteil des aktuell favorisierten BODE-Index, ist im niedergelassenen Bereich nur begrenzt durchführbar. Es fehlt meist an Platz, das Personal muss gut geschult sein und den Patienten fortlaufend anspornen. Bei wie- derholter Durchführung zeigt sich ein Lerneffekt, der einer Gehstrecke von 50-80m entsprechen kann [73].

Bei Patienten mit schwerer COPD ist ein erniedrigter Body MassIndex (unter $20 \mathrm{~kg} / \mathrm{m}^{2}$ ) ein signifikanter Risikofaktor für die Letalität [74]. Ein erhöhtes C-reaktives Protein im Serum findet man häufig auch in der stabilen Krankheitsphase, und es ist mit einer höheren Mortalität assoziiert [75]. Fragebogen zur Lebensqualität wie SF-36 oder SGRQ verursachen einen Zeitaufwand von 10-30 Minuten, bis der Patient sie ausgefüllt hat. Dies ist in der Arztpraxis zwar im Rahmen von Studien akzeptabel, nicht jedoch in der Routineversorgung.

Bei akuter Exazerbation hat sich die optische Kontrolle des Sputums auf Purulenz zu 94\% als sensitiv und in $77 \%$ als spezifisch für das Vorliegen einer bakteriellen Ursache erwiesen [76].

\section{Diskussion \\ $\nabla$}

Es gibt eine Reihe neuer Publikationen zur Frage, welche Outcome-Parameter am besten für COPD-Studien geeignet sind. Vestbo u. Mitarb. [77] haben in 2008 das Protokoll der ECLIPSEStudie zur Identifikation von prädiktiven Surrogatparametern bei COPD veröffentlicht und stellten fest, dass die $\mathrm{FEV}_{1}$ der am besten dokumentierte Beurteilungsparameter ist. In der ECLIPSE-Studie werden zahlreiche weitere Parameter über 3 Jahre auf ihre Eignung als prädiktive Surrogatparameter untersucht, da die Aussagekraft der $\mathrm{FEV}_{1}$ im Hinblick auf die Prognose und die Differenzierung unterschiedlicher Formen der COPD begrenzt ist.

Gillissen u. Mitarb. [2] haben dargestellt, welche Schwellenwerte für die Bewertung unterschiedlicher Parameter bei COPD notwendig sind, um als Erfolgsparameter einer Intervention gelten zu können. Als geeignete Parameter werden benannt: $\mathrm{FEV}_{1}$, submaximaler Belastungstest, 6-Minuten-Gehtest, Transition-Dyspnoe-Index (TDI), Borg-Skala, Visual Analog-Skala, St. George's Respiratory Questionnaire, Chronic Respiratory Questionnaire (CRQ), Quality of well-being-Skala und Exazerbationen.

Die umfassendste Darstellung der Outcome-Parameter bei COPD-Studien der ATS/ERS-Task Force [3] gelangt ebenfalls zu dem Schluss, dass Veränderungen der $\mathrm{FEV}_{1}$ nicht gleichbedeutend sind mit Änderungen von Dyspnoe, Leistungsfähigkeit oder Lebensqualität. Empfohlen wird neben der Bestimmung der $\mathrm{FEV}_{1}$ die Messung weiterer Funktionsparameter, wie FVC, IC/ TLC und die Erfassung von Dyspnoe, Gesundheitszustand, Lebensqualität und körperlicher Leistungsfähigkeit. Häufigkeit und Schweregrad von Exazerbationen werden als weitere wichtige COPD-Parameter bewertet. Mortalität, Dyspnoe und Lebensqualität werden als die bedeutendsten und belastbarsten Outcome-Parameter bezeichnet.

\section{Schlussfolgerungen \\ $\checkmark$}

Diese neueren Ansätze zeigen, dass bisher keine abschließende Antwort darauf gegeben werden kann, welcher einzelne Parameter als Studienendpunkt für eine globale Bewertung von Therapieeffekten bei COPD besonders geeignet ist. Aus den vorliegenden Daten ergeben sich zwar eine Reihe geeigneter Variablen für die Beurteilung der COPD, jedoch wurde bisher kein Parameter so gut evaluiert, dass er als Ersatz für die $\mathrm{FEV}_{1}$ als Standardparameter für die Beurteilung und Prognose der COPD dienen könnte. 
Institute

${ }^{1}$ Ehem. Direktor der Klinik Schillerhöhe, Gerlingen/Stuttgart

2 Robert Koch-Klinik, Thoraxzentrum des Klinikums St. Georg, Leipzig

${ }^{3}$ Klinik für Pneumologie und Intensivmedizin, Klinikum Lüdenscheid

${ }^{4}$ Krankenhaus Großhansdorf, Zentrum für Pneumologie und Thoraxchirurgie,

Großhansdorf, Lehrstuhl für Innere Medizin-Pneumologie, Großhansdorf,

Universität zu Lübeck

Praxis Remscheid

${ }^{6}$ Pneumologische Klinik Waldhof Elgershausen, Greifenstein/Hessen

Klinik Donaustauf, Universität Regensburg

${ }^{8}$ Berufsgenossenschaftliches Universitätsklinikum Bergmannsheil GmbH,

Medizinische Klinik III - Pneumologie, Allergologie, Schlaf- und Beatmungsmedizin, Bochum

${ }^{9}$ Medizinisch-wissenschaftliches Publizieren, Hannover

${ }^{10}$ Ruhrlandklinik Essen

${ }^{11}$ Krankenhaus Bethanien Moers, Med. Klinik III, Schwerpunkt Pneumologie,

Allergologie, Zentrum für Schlaf- und Beatmungsmedizin

${ }^{2}$ Abt. Pneumologie, Medizinische Hochschule Hannover

${ }^{3}$ Medizinische Klinik I, Klinikum Fürth

\section{Literatur}

1 Gross NJ. Outcome measurements in COPD, are we schizophrenic? Chest 2003; 123: $1325-1327$

2 Gillissen A, Buhl R, Kardos P et al. Studienendpunkte bei der chronischobstruktiven Lungenerkrankung (COPD): “Minimal Clinically Important Difference". Pneumologie 2008; 62: 149-157

3 Cazzola M, MacNee W, Martinez F-J et al. ATS/ERS Task Force on outcome of COPD. Outcomes for COPD pharmacological trials: from lung function to biomarkers. Eur Respir J 2008; 31: 416 - 468

4 Franciosi LG, Page CP, Celli BR et al. Marker of disease severity in chronic obstructive pulmonary disease. Pulm Pharmacol Ther 2006; 19: $189-199$

5 Rabe KF, Hurd S, Anzueto A et al. Global Initiative for Chronic Obstructive Lung Disease. Global strategy for the diagnosis, management, and prevention of chronic obstructive pulmonary disease: GOLD executive summary. Am J Respir Crit Care Med 2007; 176: 532 - 555

6 Celli BR, Halbert RJ, Isonaka S et al. Population impact of different definitions of airway obstruction. Eur Respir J 2003; 22: 268-273

7 Calverley PM, Burge PS, Spencer $S$ et al. Bronchodilator reversibility testing in chronic obstructive pulmonary disease. Thorax 2003; 58 : $659-664$

8 Vandevoorde J, Verbanck S, Schuermans D et al. Forced vital capacity and forced expiratory volumen in six seconds as predictors of reduced total lung capacity. Eur Respir J 2008; 31: 391 - 395

9 Taube $C$, Holz O, Mücke $M$ et al. Airway response to inhaled hypertonic saline in patients with moderate to severe chronic obstructive pulmonary disease. Am J Respir Crit Care Med 2001; 164: 1810 - 1815

10 Cerveri I, Dore R, Corsico A et al. Assessment of emphysema in COPD: a functional and radiologic study. Chest 2004; 125: 1714-1718

11 Mohsenifar Z, Lee SM, Diaz P et al. Single-breath diffusing capacity of the lung for carbon monoxide: a predictor of $\mathrm{PaO}_{2}$, maximum work rate, and walking distance in patients with emphysema. Chest 2003; 123: $1394-1400$

12 Adams AB, Cakar N, Marini IJ. Static and dynamic pressure-volume curves reflect different aspects of respiratory system mechanics in experimental acute respiratory distress syndrome. Respir Care 2001; 46: $686-693$

13 O'Donnell DE, Flüge T, Gerken F et al. Effects of tiotropium on lung hyperinflation, dyspnoea and exercise tolerance in COPD. Eur Respir J 2004; $23: 832-840$

14 Lane DJ, Howell JB, Giblin B. Relation between airways obstruction and $\mathrm{CO}_{2}$ tension in chronic obstructive airways disease. Br Med J 1968; 3 (5620): 707 - 709

15 Fletcher EC, Donner CF, Midgren B et al. Survival in COPD patients with a daytime $\mathrm{PaO}_{2}$ greater than $60 \mathrm{~mm} \mathrm{Hg}$ with and without nocturnal oxyhemoglobin desaturation. Chest 1992; 101: 649-655

16 Oga T, Nishimura K, Tsukino $M$ et al. The effects of oxitropium bromide on exercise performance in patients with stable chronic obstructive pulmonary disease. A comparison of three different exercise tests. Am J Respir Crit Care Med 2000; 161: 1897 - 1901

17 Oga T, Nishimura $K$, Tsukino $M$ et al. Analysis of the factors related to mortality in chronic obstructive pulmonary disease: role of exercise capacity and health status. AJRCCM 2003; 167: 544- 549
18 Palange $P$, Antonucci $R$, Valli $G$. Exercise testing in the prognostic evaluation pf patients with lung and heart diseases. In: Ward SA, Palange P (eds). Clinical exercise testing. Eur Respir Mon 2007; 40: 195-207

19 Bernard S, LeBlanc P, Whittom F et al. Peripheral muscle weakness in patients with chronic obstructive pulmonary disease. Am J Respir Crit Care Med 1998; 158: 629-634

20 Ottenheijm CA, Heunks LM, Sieck GC et al. Diaphragm dysfunction in chronic obstructive pulmonary disease. Am J Respir Crit Care Med 2005; 172: 200-205

21 Simonneau G, Galiè N, Rubin LJ et al. Clinical classification of pulmonary hypertension. J Am Coll Cardiol 2004; 43: 5S - 12S

22 Thabut G, Dauriat G, Stern JB et al. Pulmonary hemodynamics in advanced COPD candidates for lung volume reduction surgery or lung transplantation. Chest 2005; 127: 1531 - 1536

23 Kessler R, Faller M, Weitzenblum E et al. "Natural history" of pulmonary hypertension in a series of 131 patients with chronic obstructive lung disease. Am J Respir Crit Care Med 2001; 164: 219-224

24 Naeije $R$. Pulmonary hypertension and right heart failure in COPD. Monaldi Arch Chest Dis 2003; 59: 250-253

25 Kessler R, Faller M, Fourgaut $G$ et al. Predictive Factors of Hospitalization for Acute Exacerbation in a Series of 64 Patients with Chronic Obstructive Pulmonary Disease. Am J Respir Crit Care Med 1999; 159: $158-164$

26 Holverda S, Bogaard HJ, Groepenhoff H et al. Cardiopulmonary Exercise Test Characteristics in Patients with Chronic Obstructive Pulmonary Disease and Associated Pulmonary Hypertension. Respiration 2007 Oct 25; [Epub ahead of print]

27 DeMarco FJ Jr, Wynne JW, Block AJ et al. Oxygen desaturation during sleep as a determinant of the "Blue and Bloated" syndrome. Chest 1981; 79: 621 - 625

28 Sanders $M H$, Newman AB, Haggerty $C L$ et al. Sleep Heart Health Study. Sleep and Sleep-disordered Breathing in Adults with Predominantly Mild Obstructive Airway Disease. AJRCCM 2003; 167: 7 - 14

29 McNicholas WT, Calverley PMA, Lee A et al. Tiotropium Sleep Study in COPD Investigators. Long-acting inhaled anticholinergic therapy improves sleeping oxygen saturation in COPD. Eur Respir J 2004; 23: $825-831$

30 Marin JM, Carrizo SJ, Vicente E et al. Long-term cardiovascular outcomes in men with obstructive sleep apnoea-hypopnoea with or without treatment with continuous positive airway pressure: an observational study. Lancet 2005 Mar 19-25; 365 (9464): 10461053

31 Lavie $P$, Herer $P$, Lavie $L$. Mortality risk factors in sleep apnoea: a matched case-control study. J Sleep Res 2007; 16: 128 - 134

32 Taube C, Lehnigk B, Paasch K et al. Factor analysis of changes in dyspnea and lung function parameters after bronchodilation in Chronic Obstructive Pulmonary Disease. Am J Respir Crit Care Med 2000; 162: $216-220$

33 Schlecht NF, Schwartzman K, Bourbeau J. Dyspnea as clinical indicator in patients with chronic obstructive pulmonary disease. Chron Respir Dis 2005; 2: $183-191$

34 Vincken W, Noord JA van, Greefhorst APM et al. Dutch/Belgian Tiotropium Study Group. Improved health outcomes in patients with COPD during 1 yr's treatment with tiotropium. Eur Respir J 2002; 19: 209 216

35 Casaburi R, Mahler DA, Jones PW et al. A long-term evaluation of oncedaily tiotropium in chronic obstructive pulmonary disease. Eur Respir J 2002; 19: $217-224$

36 O'Donnell DE, Voduc $N$, Fitzpatrick $M$ et al. Effect of salmeterol on the ventilatory response to exercise in chronic obstructive pulmonary disease. Eur Respir J 2004; 24: 86 - 294

37 Hodder RV, White RJ, Menjoge SS et al. Effectiveness of Tiotropium or Salmeterol in COPD patients receiving inhaled steroids. Am J Respir Crit Care Med 2002; 165: A228

38 Jones $P W$. Interpreting thresholds for a clinically significant change in health status in asthma and COPD. Eur Respir J 2002; 19: 398 - 404

39 Calverley PM, Boonsawat W, Cseke Z et al. Maintenance therapy with budesonide and formoterol in chronic obstructive pulmonary disease. Eur Respir J 2003; 22: 912 - 919

40 Jones PW, Edin HM, Anderson J. Salmeterol/Fluticasone propionate combination improves health status in COPD patients. Am J Resp Crit Care Med 2002; 165: A111

41 Aaron SD, Vandenheen KL, Fergusson D et al. Canadian Thoracic Society/Canadian Respiratory Clinical Research Consortium. Tiotropium in combination with placebo, salmeterol, or fluticasone-salmeterol for 
treatment of chronic obstructive pulmonary disease. Ann Intern Med 2007; 146: $545-555$

42 Vijayasaratha $K$, Stockley RA. Reported and unreported exacerbations of COPD: analysis by diary cards. Chest 2008; 133: 34-41

43 Burge S, Wedzicha JA. COPD exacerbations: definitions and classifications. Eur Respir J 2003; 21: 46s - 53s

44 Aaron SD, Fergusson D, Marks GB et al. Canadian Thoracic Society/Canadian Respiratory Clinical Research Consortium. Counting, analyzing and reporting exacerbations of COPD in randomized, controlled trials. Thorax 2008; 63: 122 - 128

45 Cote CG, Dordelly LJ, Celli BR. Impact of COPD exacerbations on patientcentered outcomes. Chest 2007; 131: 696- 704

46 Soler-Cataluña JJ, Martínez-García MÁ, Sánchez PR et al. Severe acute exacerbations and mortality in patients with chronic obstructive pulmonary disease. Thorax 2005; 60: 925-931

47 Niewoehner DE, Lokhnygina $Y$, Rice $K$ et al. Risk indexes for exacerbations and hospitalizations due to COPD. Chest 2007; 131: 20-28

48 Ringbaek T, Seersholm N, Viskum K. Standardised mortality rates in females and males with COPD and asthma. Eur Respir J 2005; 25: $891-895$

49 Hansell AL, Walk JA, Soriano JB. What do chronic obstructive pulmonary disease patients die from? A multiple cause coding analysis. Eur Respir J 2003; 22: 809-814

50 Ringbaek T, Viskum K. Is there any association between inhaled ipratropium and mortality in patients with COPD and asthma? Respir Med 2003; 97: 264 - 272

51 Zielinski J, MacNee W, Wedzicha J et al. Causes of death in patients with COPD and chronic respiratory failure. Monaldi Arch Chest Dis 1997; 52: $43-47$

52 Gudmundsson G, Gislason T, Lindberg E et al. Mortality in COPD patients discharged from hospital: the role of treatment and co-morbidity. Respir Res 2006; 16 (7): 109

53 Curkendall SM, Lanes S, de Luise C et al. Chronic obstructive pulmonary disease severity and cardiovascular outcomes. Eur J Epidemiol 2006; 21: $803-813$

54 Sin $D D, W u$ L, Anderson J et al. Inhaled corticosteroids and mortality in chronic obstructive pulmonary disease. Thorax 2005; 60: 992 - 997

55 Calverley PMA, Anderson JA, Celli B et al. TORCH investigators. Salmeterol and Fluticasone Propionate and Survival in Chronic Obstructive Pulmonary Disease. N Engl J Med 2007; 356: 775 - 789

56 Kesten S, Jara M, Wentworth C et al. Pooled clinical trial analysis of tiotropium safety. Chest 2006; 13: 1695-1703

57 Celli BR, Cote CG, Marin JM et al. The body-mass index, airflow obstruction, dyspnea, and exercise capacity index in chronic obstructive pulmonary disease. N Engl J Med 2004; 350: 1005 - 1012

58 Casanova C, Cote C, de Torres JP et al. Inspiratory-to-total lung capacity ratio predicts mortality in patients with chronic obstructive pulmonary disease. Am J Respir Crit Care Med 2005; 171: 591 - 597

59 Holguin F, Folch E, Redd SC et al. Comorbidity and mortality in COPDrelated hospitalizations in the United States, 1979 to 2001. Chest 2005; 128: 2005 - 2011

60 Young RP, Hopkins $R$, Eaton TE. Forced expiratory volume in one second: not just a lung function test but a marker of premature death from all causes. Eur Respir J 2007; 30: 616-622
61 Garcia-Aymerich J, Lange P, Benet $M$ et al. Regular physical activity reduces hospital admission and mortality in chronic obstructive pulmonary disease: a population based cohort study. Thorax 2006; 61: $772-$ 778

62 Watz H, Waschki B, Boehme C et al. Extrapulmonary effects of chronic obstructive pulmonary disease on physical activity: a cross-sectional study. Am J Respir Crit Care Med 2008; 177: 743-745

63 Wewel AR, Gellermann I, Schwertfeger I et al. Intervention by phone calls raises domiciliary activity and exercise capacity in patients with severe COPD. Respir Med 2008; 102: 20-26

64 Vogelmeier C, Buhl R, Criée CP et al. Leitlinie der Deutschen Atemwegsliga und der Deutschen Gesellschaft für Pneumologie und Beatmungsmedizin zur Diagnostik und Therapie von Patienten mit chronisch obstruktiver Bronchitis und Lungenemphysem (COPD). Pneumologie 2007; 61: e1 - e40

65 Nationale Versorgungsleitlinie COPD NVL Deutscher Ärzte-Verlag, 2007

66 Casaburi R, Kufkafka D, Cooper CB et al. Improvement in exercise tolerance with the combination of tiotropium and pulmonary rehabilitation in patients with COPD. Chest 2005; 127: 809-817

67 Decramer M, Gosselink R, Bartsch P et al. Effect of treatments on the progression of COPD: report of a workshop held in Leuven, 11-12 March 2004. Thorax 2005; 60: 343-349

68 Dhein Y, Birkenmaier A, Otte B et al. Evaluation of a structured education programme (AFBE) for patients with mild to moderate COPD under outpatient conditions. Am J Respir Crit Care Med 2002; 165: A420

69 Tirimanna PR, Den Otter JJ, Van Schayck CP et al. Evaluation of the suitability of weekly peak expiratory flow rate measurements in monitoring annual decline in lung function among patients with asthma and chronic bronchitis. Br J Gen Pract 1996; 46: 15 - 18

70 Glaab T, Banik N, Rutschmann OT et al. National survey of guidelinecompliant COPD management among pneumologists and primary care physicians. COPD 2006; 3: 141 - 148

71 Nishimura K, Izumi T, Tsukino $M$ et al. Dyspnea is a better predictor of 5 -year survival than airway obstruction in patients with COPD. Chest 2002; 121: $1434-1440$

72 Burdon JG, Juniper EF, Killian KJ et al. The perception of breathlessness in asthma. Am Rev Respir Dis 1982; 126: 825 - 828

73 Guyatt GH, Sullivan MJ, Thompson PJ et al. The 6-minute walk: a new measure of exercise capacity in patients with chronic heart failure. Can Med Assoc J 1985; 132: 919-923

74 Landbo C, Prescott E, Lange P et al. Prognostic value of nutritional status in chronic obstructive pulmonary disease. Am J Respir Crit Care Med 1999; 160: 1856 - 1861

75 Man SF, Connett JE, Anthonisen NR et al. C-reactive protein and mortality in mild to moderate chronic obstructive pulmonary disease. Thorax 2006; 61: 849-853

76 Stockley RA. New approaches to the management of COPD. Chest 2000; 117: 58S-62S

77 Vestbo J, Anderson W, Coxson HO et al. ECLIPSE investigators. Eur Respir J 2008; 31: 869-873 